\title{
PRE-PRINT
}

\section{Ethnic and Gender Diversity, Process and Performance in Groups of Business Students in Sweden}

\author{
Timurs Umans*, Sven-Olof Collin, Torbjörn Tagesson
}

\author{
Department of Business Studies \\ Kristianstad University College \\ 29188 Kristianstad \\ Sweden
}

*Corresponding author. Timurs Umans: timurs.umans@hkr.se

Accepted for publication at Intercultural Education (2008) 


\begin{abstract}
This paper investigates the complex interrelation between ethnic and gender diversity, process and performance in groups of business students. The paper is based on an empirical survey of business students working on a complex assignment in groups of two to five in a small Swedish university. The results indicate that gender diversity leads to positive group outcomes, while ethnic diversity appears to create negative group outcomes. Intervening process variables, such as group communication, conflict and effectiveness in problem solving were not found to be influenced by diversity, or to influence group outcomes. While the non-influence of intervening variables can probably be explained by methodological difficulties, the negative outcome for ethnic diversity indicates a need to help students better manage ethnic diversity in order to reap its benefits.
\end{abstract}




\section{Introduction}

Student groups have become an integral part of the instructional process (Watson et al., 2002) and have been frequently used as an instructional method in the institutions of higher education. For instance, team learning (Michaelsen et al., 1982) and cooperative learning (Johnson and Johnson, 1992) have attracted scrutiny in recent research. With increasing cultural and gender diversity in the classroom, group assignments serve as a golden opportunity for the exploitation of this diversity that has become a reality in higher education today.

This article tries to draw conclusions on how this diversity can be assessed and managed in higher education. The aim of the study is to make methodological, theoretical, and practical contributions to research about cultural and gender diversity in learning teams (in the case of this study, the diversity due to presence of foreign students). The methodological contribution of this article is represented by the alternative operationalisation of ethnic diversity, expressed not only in terms of race, which has become a tradition in ethnic diversity research (e.g. Watson et al. 1998; McLeod and Lobel, 1992), but in terms of nationality and national origin as well as self-identification. The theoretical contribution of this article is an attempt to inquire into the intervening group processes, a fairly unexplored area because of its presumed complexity. A practical contribution of this paper is the guidance on how to manage cultural diversity both from the educators' and students' perspective. The structure of the paper is as follows: a review of the literature on the ethnic and gender diversity in teams and the interrelations with group process and outcomes is followed by sections on method, analysis, subsequent conclusions and suggestions for future research.

\section{Literature review}

\section{Ethnic Diversity in Teams}

Scholars actively working within the demographic composition model, where processes remain in the 'black box' and the direct connection between diversity and outcomes is investigated, claim that ethnically diverse teams are able to perform better in turbulent and complex environments (Shaw, 1981), where the group members' cultural diversity serves as a drive for flexibility, and receptiveness to environmental change and turbulence (Wagner, 1995). Ethnic diversity in groups and 
teams tend to lead to a higher quality of decisions (McCarrey, 1988) and ideas (McLeod and Lobel, 1992). This positive outcome streams from the variety of viewpoints provided by differences in cultural backgrounds, which subsequently indicates that ethnically diverse groups outperform ethnically homogeneous groups (Buller, 1986).

In studies of learning teams, Watson and colleagues (Watson and Kumar, 1992; Watson et al., 1993) generally came to the conclusion that during the early stages of team life, ethnically diverse teams perform less effectively than non-diverse teams (Watson et al., 1998). However, over time not only do these differences wash out, but they may become an asset for team results (Wagner, 1995). These findings come from teams that work together and receive feedback on particular team tasks (Watson et al., 2002). According to Michaelsen and Watson (1987), at the end of the learning team's life cycle, the advantage of multiple viewpoints due to cultural diversity has been shown to result in higher performance, which is in line with the demographic composition model. By working within a limited time frame, and having the results of their work evaluated and counted towards their final grades, learning teams are put into complex environments. Moreover, the tasks learning teams usually perform are complex in their nature due to demands in terms of creativity and analytical approach that are required in order to attain a higher grade on the team assignment. Based on the foregoing discussion, we propose that:

Proposition 1: Increasing degree of ethnic diversity in a learning team will have a positive influence on team performance later in the team project life cycle when handling complex problem solving.

Researchers on intervening models, where processes are being extracted from the black box for study, have been much more divided regarding the effects of ethnic diversity on both process and performance of teams. From one side, it is claimed that culturally diverse teams offer diversity of values, resulting in effective group discussions that ultimately lead to enhanced group performance (Hofstede, 1984; McCarrey, 1988). Moreover, cultural diversity of groups leads to more cooperative choices (Cox et al., 1991) and better performance in comparison with homogeneous groups in identifying perspectives of the problems and generating solution 
alternatives (Watson et al., 1993). The voices from the other side of the argument, however, are louder and supported by more empirical evidence. Researchers who claim negative effects of cultural diversity on process and outcomes maintain that cultural diversity in teams results in interpersonal problems and communication difficulties (Triandis, 1960), misunderstandings and threats to team cohesiveness (O’Reilly, Cardwell, and Barnett, 1989). Generally, many researchers have come to the conclusion that cultural diversity has a negative effect on processes taking place within the team such as communication (Triandis, 1960) and result in emotional (Pelled, 1996) and competitive conflicts (Kirchmeyer and Cohen, 1992). These conditions in turn lead to lower performance when compared to the ethnically homogeneous learning teams (Watson et al. 1993), since ethnically diverse team members are likely to have fewer shared experiences, less in common, and more difference of opinion, usually leading to conflict within the team (Pfeffer, 1983) However, several authors have claimed that ethnically diverse teams will be superior to ethnically homogeneous teams in their effectiveness in complex problem solving (Shaw, 1983).Hence, this leads us to the following three propositions:

P2: Increasing degree of ethnic diversity in learning teams will have a negative effect on communication.

P3: Increasing degree of ethnic diversity in learning teams will have a positive effect on the degree of conflict.

P4: Increasing degree of ethnic diversity in learning teams will have a positive impact on the effectiveness in complex problem solving.

It is argued that efficiency of communication as well as effectiveness in complex problem solving will have a positive influence on team performance (Smith et al., 1994). Eisenhardt and Bourgeois (1988) found that teams that experience internal strife or conflict decline in performance. This is supported by findings of Jehn (1997) who claims that conflict constrains effectiveness in creative problem solving and influences performance negatively. Moreover, Elron (1997) in her study of top management teams found that small teams with less social cohesion and ineffective communication will be negatively related to group performance. Thus, we propose: 
P5: Effective communication in learning teams will have a positive effect on teams' performance.

P6: Increasing degree of conflict in learning teams will have a negative effect on teams' performance.

P7: Effective complex problem solving in learning teams will have a positive effect on performance.

\section{Gender diversity in learning teams}

It has also been noted that group and outcome are influenced by the complex composition of diversity dimensions, rather than isolated dimensions of diversity (Jackson et al., 2003), and the team and organisational outcomes may be determined by the configuration of team members' demographic and/or identity profiles (Frable, 1997). Gender, as one of the demographic variables, has been accorded special attention in research due to the increasing number of women entering organisations, including the increase of female students entering institutions of higher education. For instance, a meta-analysis by Wood (1987) showed that mixed-gender groups tended to perform better than homogeneous-gender groups. It was also noted that the behavioural differences between men and women in mixed-gender groups may be especially influenced by a group’s gender balance (e.g. Johnson and Schulman, 1989) Gender diversity influences the team's performance in higher education since females tend to perform better than males (Byrne, Flood and Willis, 2001), implying that with an increased number of females in a group, the group might perform better. However, a team homogeneous in gender will be restricted in input variety and opinion diversity, thus reducing performance in a complex assignment or/and complex environment (Dess and Beard, 1984). Therefore, it is not the proportion of men or women, but the mix that influences performance. Thus, we propose that

P8: Increasing degree of gender diversity in learning teams will increase the performance of the group when subjected to complex problem solving.

Results have been mixed on the influence of gender composition on team process (Watson et al., 1998). However, research indicates that gender diversity tends to 
affect behaviour, communication, and individual experience within groups, while it does not affect group performance per se (Smith-Lovin and Brody, 1989). According to Wood (1987) gender-balanced groups would have more positive interaction, including communication and conflict reduction, compared to predominantly male or predominantly female teams. Moreover, experimental studies by Stringer (1995) have shown that gender-balanced groups are more consensus-seeking, a characteristic that improves communication within the group and reduces conflict. Kanter's study of minority/majority grouping shows that groups with a 'token' member (e.g. one female and three males, or one male and three females) would likely be more subject to stereotyping and marginalization than gender-balanced groups (1977). When it comes to gender, it is believed that men and women have different perspectives on how tasks should be performed and goals achieved (Stringer, 1995). According to Mannix and Neale (2005), the extent to which groups are diverse in their perspectives positively influences effectiveness in problem solving. Based on the foregoing discussion we propose:

P9: Increasing degree of gender diversity in learning teams will have a positive effect on communication.

P10: Increasing degree of gender diversity in learning teams will have a negative effect on the degree of conflict.

P11: Increasing degree of gender diversity in learning teams will have a positive impact on the effectiveness in complex problem solving.

\section{Method}

\section{Participants}

The sample consisted of 102 participants (57 males, 56\% and 45 Females, 44\%) who enrolled in the corporate strategy course, which is a part of international business programme, at a university college in southern Sweden. The 29 self-arranged teams each consisted of two to five individuals working on the case study, representing $20 \%$ of the final individual grade, which made the results of teamwork important to each course participant. The course was included in an international business programme 
offered to foreign exchange students and Swedish students. Hence, the class was composed of students from Sweden and other countries.

The complexity of the environment was achieved (a) through combination of students both from abroad and from different study programmes within the department and who had never studied together before; (b) by a tight time schedule; and (c) by the importance of the case study for students' final grades. Completion of the group assignment took approximately 15 hours of group work.

\section{Measures}

\section{Dependent variable}

Team performance was measured based on a written analysis of the case study. Students were evaluated on a number of criteria such as case presentation, theoretical model application, analysis, conclusion and use of external sources. Maximum possible grade for the case was 20 points. In order to insure non bias of the evaluation process, the names of group members were removed, so that only group number and number of students in the group were known. Thus the evaluator could not discover the identity of the people in the group.

\section{Mediating variables}

Process variables were measured on a self-reporting basis, where respondents were asked to mark on a 7-point Likert scale the degree of a certain process, such as effectiveness in problem solving. Communication in the group was observed through three questions on constructiveness of discussion, informality of communication and effectiveness of communication flow. A reliability test of the three variables indicated acceptable reliability (Cronbach's alpha $=0.70$ ). They were summed and divided by three in order to make them comparable with the other two process variables. Conflict was measured by asking the respondents to indicate the degree of conflict present in the learning team while working on the assignment. Effectiveness in problem solving was measured by self-evaluation of the group members. In order to construct a total degree of communication, conflict and effectiveness in problem solving for each group, a proportional approach was used, where the sum of each team member's answers was proportionate to the highest possible degree of these three process variables per group. This approach was based on Blau's seminal work, Inequality and 
Heterogeneity (1977), which argues that proportion of majority/minority membership in the group helps to determine the quality of relations between demographically different groups. The strength of the proportional approach, according to Mannix and Neale (2005), is that it allows a focus on relative differences, recognizing that a diverse group with a minority of one is qualitatively different from a group with more balanced proportions.

\section{Independent variables}

Ethnic diversity was measured as a combination of different variables such as nationality, ethnic self-identification, country of birth, native language, native language and country of birth of both the mother and father. This particular measurement of ethnic identity differs from previously used methods (e.g. Cox, et al., 1991; Watson et al., 1998) where one particular variable such as race or nationality was taken as equal to ethnicity. Since ethnic identity is believed to be a multifaceted phenomenon (Campbell, 2000), it is beneficial to assess ethnic diversity in terms of different aspects (Hecht, 1993). The complexity of ethnic identity is diminished by putting it into just one self-identification variable (e.g. race) while researchers acknowledge that ethnic identity could be conceived in terms of four frames: personal, enactment, relationship and communal. (Stephan and Stephan, 2000). Thus,

the degree of ethnic diversity was measured as a proportion of people dissimilar to others in the group (Blau, 1977) with respect to the ethnicity dimensions mentioned earlier. Gender was measured by self-identification by the group members and as a proportion of people dissimilar to others, in terms of gender, in the group.

\section{Control variable}

Size of the group was considered to be a control variable. When evaluating the performance of the group, the number of students who influenced the performance had to be considered. A group with more students would perform better (ceteris paribus) and receive a higher grade if the evaluator did not consider group size. Thus, if the evaluator is not capable of considering the influence of size, we expect a positive relationship between size and performance. 


\section{Analytical method}

We use a linear regression method analysing the relationship between the independent variables, the mediating variables and the dependent variable in three different models (Allison, 1999).

\section{Analysis}

The descriptive statistics of the variables are presented in Table 1

Insert Table 1 about here

The values for dependent variable Grade show that the groups received 15.04 points on average with rather small deviation, thus indicating a compressed grading (minimum grade given was 10 and maximum was 18). The group size, ranging from 2 to 5 , was on average 4.6, and the deviation indicates that most of the groups contained 3 or 4 students. Gender diversity varied between 0 and 0.5 , and the average of 0.126 indicates rather low gender diversity. In fact, about 16 of 27 groups had no gender diversity at all. Ethnic diversity was measured as national diversity, on which we will comment later in the paper. National diversity was higher due to a higher variation of nationality than of gender. The process variables show high average on communication and on effectiveness in problem solving, but lower on conflict.

Inspecting the correlation matrix, grade appears to correlate positively with gender diversity, but negatively with national diversity. The process variables do not appear to correlate with grade, except for the positive correlation of effectiveness in problem solving. Thus, our proposition of gender (P8) appears to be supported, while national diversity proposition appears to be contrary to expectation (P1). Process variables appear not to be influenced by diversity and appear not to influence performance (P2, P3, P4 - ethnic diversity influences on process propositions - not supported; P9, P10, P11 - gender diversity influences on process - not supported; P5, P6, P7 - process influences on performance - not supported). Our control variable, group size, is not correlated to grade, which indicates that the grader considered group size when evaluating the performance of the group. 
The correlation matrix indicates that we can expect collinearity problems for the process variables since they are highly correlated. No other independent variable appears to present collinearity problems.

Our first analysis (Table 2) tests the black-box regression model, where the diversity measures are directly correlated with the performance, i.e. the grade.

Insert Table 2 about here

The model is highly significant, being able to explain more than half of the variance. Gender diversity indicates support of P8 that increased gender diversity will increase performance. It should be noted that when including proportion of gender diversity, the gender variable showed no significant correlation, i.e. it is not the gender proportion, but the diversity that influences performance. Ethnic diversity, measured according to nationality (i.e. National diversity), however, negatively influenced performance, quite contrary to P1 stating a positive correlation between ethnic diversity and performance. We considered several other measurements of ethnic diversity, such as country of birth, native language and parents' national identity and language as well as students' self-identification concerning the nation they mostly associated themselves with; but with these measurements the diversity variable was not significant (analyses available on request). We will return to the issue of measurement in the concluding discussion.

Next, we analysed the relationship between the independent variables of diversity and the intermediating process variables of communication, conflict and effectiveness of problem solving. But we present only the regression model with the highest $\mathrm{R}^{2}$ in Table3.

Insert Table 3 about here 
As is evident in Table 3, none of the diversity variables are correlated with the process variable, as the results in all the other regression analyses The only slight indication that can be found in all regressions on process variables is the somewhat weak significance (in this model the significance level is 0.11 ) of national diversity that correlates negatively with effectiveness in problem solving (P4). Thus, there is no support in our analyses for the proposition that diversity influences the process (P2, P3, P4 and P9, P10, P11 - not supported).

The third model is the relationship between the process variables and the performance variable of the grade. Since we found that the process variables created collinearity problems, we could not analyze any model including all three process variables. We present here the most interesting model, but all other models present outcomes that lead to the same conclusions to be drawn from the presented analysis, as can be seen in Table 4. (Omitted analyses can be obtained from the authors)

Insert Table 4 about here

The model does not strongly explain the variation in performance, and the significance is weaker than the model with a direct link between diversity and performance. The model is only weakly significant, and the whole burden of explanation is on the effectiveness in problem solving variable. Thus, we cannot make a strong case for process influencing performance.

Summarising the analyses we have found indication that diversity influence performance: gender diversity positively, and national diversity negatively. Only very small indications could be found in support of the intermediating model of process, indicating a negative relationship between national diversity and effectiveness in problem solving, with problem solving being positively correlated with performance. 


\section{Discussion}

The overall goal of this paper has been to inquire into the relationships between ethnic diversity in learning teams, team processes and team outcomes. The paper has followed two paths of inquiry. Firstly, we tested the demographic composition model, where we explored a direct relationship between ethnic diversity in learning teams and its influence on group performance, leaving the process variables in the black box. Secondly, we tested the intervening process model, where the relationship between ethnic diversity in learning teams is assumed to influence processes within the team; in this paper, the processes investigated were communication, conflict and effectiveness in problem solving. Then, the processes in the learning teams were assumed to influence team performance. Our analyses indicate that a demographic composition model, where process remains in the black box, has a better explanatory power with regard to the influences of both gender and ethnic diversity on performance, while the intervening model produces no consistent results.

Even though we have attempted to use a multidimensional approach to ethnicity as pleaded by some researchers (e.g. Millikin and Martins, 1996), it was only national diversity that showed a significant correlation to learning team performance. The finding indicates that objective measurements of ethnicity could be more valid, and that the environment of the individual has more influence on the ethnic identity of the individual than does the ethnic origin of the individual's father and mother. The simplistic questions in the questionnaire could be another explanation of why we did not manage to capture other possible diversity variables. It should be noted that results of this paper are based on the national diversity, achieved through the mixture of Swedish and foreign students in the course, and not on ethnic diversity expressed in terms of native language, parental ethnic background, and country of origin.

As for the influence of gender diversity on team performance, our analysis supports our proposition that with an increase of gender diversity there will be an increase in performance. This is in line with findings in gender diversity research, where it is believed that differences in task orientation between men and women increase performance of gender-balanced teams (Wood, 1987). 
In contrast to our expectations, nationality as one of the measures of ethnic diversity has a negative influence on performance. What makes this result especially striking is the fact that students that participated in the survey are international business students who are supposed to have an international mindset, since the enrolment into the programme is voluntary and based on the students' supposed desire to explore and get in touch with people of different cultures. One possible reason is that nationalities represented in the teams were not very compatible with each other, when looking at them, for example, from Hofstede’s cultural dimensions (1984). There could be a possibility that collectivistic and individualistic cultures might not work well together, with individuals seeking communality or privacy, respectively, which would decrease group performance because of differences on specific cultural dimensions (Jehn and Mannix, 2001). Thus, in future studies it would be interesting to observe not merely the degree of national diversity in teams, but also the combination of different nationalities in the group, graded by their cultural dimension indexes; these could be combined into a group's total score to enable comparison with other groups, or to examine in-group behaviour and its influence on group performance. According to Ely and Thomas (2001), ethnic diversity can result in lower comparability to the outcomes of ethnically homogeneous teams' outcomes, due to the lack of training and development initiatives. It can be assumed that students in our sample were relatively inexperienced and not trained in ethnic diversity assessment, and that they lacked the understanding of problems that could arise from this diversity since the majority of them were first-year students and had not been exposed to work with students of different ethnic backgrounds prior to the course. Another explanation of the negative influence of nationality on performance could be the relatively short team life, which was only four weeks for the teams in our sample; this is supported by findings of Watson et al. (1998) and Keck (1991), who claim that diversity benefits become apparent as the group’s tenure increases.

Our expectations concerning gender and ethnic diversity in its influence on processes have not been realised. There could be several reasons for our prediction failure. Firstly, the problem could lie in the measurement of the process variables. Secondly, it can be assumed that even if the process variable and processes representing the variable were measured correctly, the problem of leading to no result may lie in the self-reporting technique employed in this research. Making an assumption that 
students can be their own observers and interpreters is very optimistic, but questionable. Thirdly, the problem may originate in our assumption based on previous research (Smith et al., 1994) that diversity has a direct influence on processes. Several authors (e.g. Ely and Thomas, 2001; Larkey, 1996) argue that the demographic diversity- process relationship could have a moderating variable such as common values and beliefs, or shared goals that would bare the instrumental importance of the mediator between the two. Thus there might be a possibility that the intervening model we attempted to test lacked the moderating variables that could influence the relationship between ethnic and gender diversity on processes. Fourthly, the problem may lie in the operationalisation of the diversity variable (Pitcher and Smith, 2001); however this is highly improbable in light of the very strong significance between national diversity and performance discovered when testing the demographic composition model.

The problems of methodology, which are believed to be the drive for the inconsistencies and non-results in diversity research (Pitcher and Smith, 2001), can also be put forward as one of the explanations for our data not showing the influence of processes on performance. Thus, one possible option, though not a panacea, could be the use of qualitative methods such as case study research. This could enable one to inquire into the black box of processes, which some researchers argue could bring new insight and better understanding of the black-box complexity and its possible correlation with group diversity and group outcomes (Ruigrok and Tacheva, 2004). A combination of interviews to identify process variables and the use of survey data to test derived process conceptualisation could be one of the ways to make studies of processes more focused and possibly allow more precise and clear definitions of process variables.

\section{Practical implications}

Our findings have several practical implications, both for students involved in multiethnic group work and for teachers using multi-ethnic groups in their courses. The first implication is that we could tell students that in order to achieve higher results they should form gender-balanced and nationally homogeneous groups. While that naturally would be an easy way to interpret our findings, nevertheless our studies 
show that diversity has to be managed since ethnic diversity is on the increase in almost every part of our society.

Accordingly, as educators we should encourage our students to form ethnically diverse teams for the sake of their future success in a diverse world. Especially keeping in mind that intercultural communication competences or in other words "ability to understand the meaning of intercultural interaction and the ability to adapt one's behaviour to these meanings in order to produce efficient behaviour" (BartelRadic, 2006, p. 651) required in today multicultural work environment are usually developed in intercultural interactions which take place in our classrooms.

In order to manage diversity from within the group, students who want to achieve higher performance in ethnically diverse groups should consider and learn to build social ties or bridges with their team members. According to Mannix and Neale (2005), the point in social bridging is to emphasize what is similar among team members rather than simply what is different. In order for national diversity in groups to improve outcomes, and to make differences work towards innovative creativity, educators might encourage ethnically diverse group members to discuss what they have in common, as well as what each team member brings to the group, all of which could promote bridging and subsequently increased performance. This idea is also supported by the findings of McGurty and Silova (2000), who suggest that social bridging in various forms helps students to overcome certain stereotypes and prejudices and increase various team outcomes. One of the more ambitious ways of dealing with diversity in learning teams and diversity in institutions of higher education is a desirable change in organizational culture, shifting from the traditional methods of dealing with diversity ('discrimination and fairness' and 'access and legitimacy') to a more progressive and learning-based view (Ely and Thomas, 2001). The learning approach to diversity can be expressed in the creation of an environment in the institutions and classrooms of higher education that would emphasize the benefits of gender and ethnic differences for organizations; for it is through differences of opinion, value and belief and through similarities in aims and taskrelated values that organizational and team ambitions of innovativeness and higher performance can be achieved. 
Culturally diverse teams are widely used by many organisations today, thus the results of the study might have practical implications for managers. This study highlights possible performance hindrances resulting from cultural diversity in teams and possible performance enhancements resulting from gender diversity teams. However, as noted in the discussion part of the paper, the negative results of ethnic diversity in team can be influenced by relatively short life-time of the learning teams under study, which might not be true for the teams employed in organisational settings, where teams usually have a longer life-span and thus have a possibility to benefit form a variety of opinions streaming form differences in ethnicity, while at the same time having enough time to overcome communication difficulties. 


\section{References}

Allison, P. D. (1999) Multiple Regression: A Primier.Pine Forge Press: Thousand Oaks, CA (US).

Bartel-Radic, A. (2006) Intercultural Learning in Global Teams. Management International Review. 46: 647-677

Blau, P. (1977) Inequality and heterogeneity. New York: Free Press.

Buller, P. F. (1986) The team building-task performance relation: some conceptual and methodological refinements. Group and Organization Studies, 10: 147-169.

Byrne, M., Flood, B. and Willis, P. (2001) The relationship between learning approaches and learning outcomes: a study of Irish accounting students. Accounting Education 11: 27-42.

Campbell, A. (2000) Cultural identity as a social construct. Intercultural Education, 11: 31-39.

Cox, T., Lobel, S. A., and McLeod, P. L. (1991) Effects of ethnic group cultural differences on cooperative and competitive behavior on a group task. Academy of Management Journal, 4: 827-847.

Dess, G. G., and Beard, D. (1984) Dimensions of organizational task environments. Administrative Science Quarterly, 29: 52-73.

Eisenhardt, K. M., and Bourgeois, L. J. III. (1988) Politics of strategic decisionmaking in high velocity environments: towards a midrange theory. Academy of Management Journal, 31: 737-770.

Elron, E. (1997) Top management teams within multinational corporations: effects of cultural heterogeneity. Leadership Quarterly, 8(4): 393-412.

Ely, R. J., and Thomas, D. A. (2001) Cultural diversity at work: the effects of diversity perspectives on work group processes and outcomes. Administrative Science Quarterly, 46: 229-273.

Frable, D. E. S. (1997) Gender, racial, ethnic, sexual, and class identities. Annual Review of Psychology, 48: 139-162.

Hecht, M. L. (1993) 2002-A research odyssey: toward the development of a communication theory of identity. Communication Monographs, 60: 76-82

Hofstede, G. (1984) Culture's consequences: International differences in workrelated values. Beverly Hills, CA: Sage.

Jackson, S. E, Joshi, A., and Erhardt, N. L. (2003) Recent research on team and organizational diversity: SWOT analysis and implications. Journal of Management, 29(6):801-830.

Jehn, K. A. (1997) A qualitative analysis of conflicts types and dimensions in organisational groups. Administrative Science Quarterly 42: 530-557.

Jehn, K. A., and Mannix, E. (2001) The dynamic nature of conflict: a longitudinal study of intragroup conflict and group performance. Academy of Management Journal, 44: 238-251.

Johnson, D. W., and Johnson, F. P. (1992) Positive interdependence: the heart of cooperative learning. Edina, NM: Interaction Book Company.

Johnson, R. A., and Schulman, G. I. (1989) Gender-role composition and role entrapment in decision-making groups. Gender and Society, 3: 355-372.

Kanter, R. (1977) Some effects of proportions on group life: skewed sex ratios and responses to token women. American Journal of Sociology, 82: 965-990.

Keck, S. L. (1997) Top management team structure: Differential effects by environmental context. Organization Science, 8: 143-156.

Kirchmeyer, C., and Cohen, A. (1992) Multicultural groups: their performance and 
reactions with constructive conflict. Group and Organization Management, 2: 153-171.

Larkey, L. K. (1996) Toward a theory of communicative interactions in culturally diverse workgroups. Academy of Management Review, 21: 463-491.

Mannix E., and Neale, M. A. (2005) What differences make a difference? Psychological Science in the Public Interest, 2: 31-55.

McCarrey, M. (1988) Work and personal values for Canadian anglophones and francophones. Canadian Psychology, 29:69-83.

McGurty, N., and Silova, I. (2000) Ethnic integration and cross-culturalism in an academic setting: the case of the Stockholm School of Economics in Riga. Intercultural Education, 11:79-91.

McLeod, P., and Lobel, S. (1992) The effects of ethnic diversity on idea generation in small groups. Paper presented at the annual meeting of the Academy of Management, Las Vegas, NV.

Michaelsen, L. K., and Watson, W. E. (1987) Teaching through an experience with giving and receiving feedback. Organizational Behavior Teaching Review, 11: 111-113.

Michaelsen, L.K., Watson, W. E., Cragin, J. P., and Fink, L-D. (1982) Team learning: a potential solution to the problems of large classes. Exchange: The Organizational Behavior Teaching Journal.

Milliken, F. J., and Martins, L. L. (1996) Searching for common threads: understanding the multiple effects of diversity in organizational groups. Academy of Management Review, 21: 402-433.

O'Reilly, C. A. III, Caldwell, D., and Barnett, W. (1989) Work group demography, social integration, and turnover. Administrative Science Quarterly, 34: 21-37.

Pelled, L. (1996) Demographic diversity, conflict and work group outcomes: An intervening process theory. Organization Science, 7: 615-631.

Pfeffer, J. (1983) Organizational demography. In L. L. Cummings and B. M. Staw (Eds.), Research in organizational behavior, vol.5: 295-357. Greenwich, CT: JAI Press.

Pitcher, P., and Smith, A. (2001). Top management team heterogeneity: Personality, power, and proxies. Organization Science, 12: 1-18.

Ruigrok, W., and Tacheva, S. (2004) Top management team diversity: Review of research gaps and methodologies. Paper presented at the 20th European Group of Organization Studies (EGOS) conference, Ljubljana, Slovenia, July 2004

Shaw, M. E. (1981) Team Dynamics: The Psychology of Small Team Behavior. 3rd edition, New York: McGraw-Hill.

Shaw, M. E. (1983) Group Composition. In H.J. Blumberg, A. P. Hare, V. Kent, and M.F. Davies (Eds.), Small group and social interaction, vol. 1. Chichester, England: Wiley.

Smith-Lovin, L., and Brody, C. (1989) Interruptions in group discussion: The effects of gender and group composition. American Sociological Review, 54: 425-435.

Smith, K.G., Smith, K.A., Olian, J.D., Sims, H.P. Jr, O’Bannon, D.P. and Scully, J.A. (1994) Top management team demography and process: the role of social integration and communication. Administrative Science Quarterly, 39: 412- 438.

Stephan, C. W., and Stephan. S. G. (2000) The measurement of racial and ethnic identity. International Journal of Intercultural Relations, 24: 541-552.

Stringer, D. M. (1995) The role of women in workplace diversity consulting. Journal of Organisational Change, 1: 44-51. 
Triandis, H. C. (1960) Cognitive similarity and communication in a dyad. Human Relations, 13: 175-183.

Wagner, J. A. III. (1995) Studies of individualism-collectivism: Effect in cooperation in groups. Academy of Management Journal, 38: 152-172.

Watson, W. E., Johnson, L., and Zgourides, G. D. (2002) The influence of ethnic diversity on leadership, group process, and performance: an examination of learning teams. International Journal of Intercultural Relations, 26: 1-16

Watson, W. E., Johnson, L., Kumar, K., and Critelli, J. (1998) Process gain and process loss: comparing interpersonal processes and performance of culturally diverse and non-diverse teams across time. International Journal of Intercultural Relations, 22: 409-430.

Watson, W. E., Johnson, L., and Merritt, D. (1998) Team orientation, selforientation, and diversity in task groups. Group and Organization Management, 23: 161-188.

Watson, W. E., and Kumar, K. (1992) Factors associated with differences in decision making regarding risk taking: A comparison of culturally homogeneous and culturally heterogeneous groups. International Journal of Intercultural Relations, 16: 53-65.

Watson, W. E., Kumar, K., and Michaelsen, L. K. (1993) Cultural diversity's impact on interaction process and performance: Comparing homogeneous and diverse task groups. Academy of Management Journal, 36: 590-602.

Wood, W. (1987) Meta-analytic review of sex differences in group performance. Psychological Bulletin, 102: 53-71. 
Table 1.

Descriptive statistics and correlation matrix $(n=27)$

\begin{tabular}{|c|c|c|c|c|c|c|c|c|}
\hline Variable & Mean & Std dev & 2 & 3 & 4 & 5 & 6 & 7 \\
\hline 1. Grade & 15.04 & 2.609 & 0.15 & $0.42^{*}$ & $-0.58^{* *}$ & 0.08 & 0.08 & $0.38^{*}$ \\
\hline 2. Group size & 3.56 & 0.641 & $\mathbf{X}$ & 0.07 & 0.07 & -0.04 & 0.24 & -0.05 \\
\hline 3. Gender diversity & 0.126 & 0.162 & & $\mathbf{X}$ & 0.10 & -0.09 & -0.16 & 0.16 \\
\hline 4. National diversity & 0.287 & 0.352 & & & $\mathbf{X}$ & -0.10 & -0.06 & 0.30 \\
\hline 5. Communication & 0.829 & 0.104 & & & & $\mathbf{X}$ & $-0.61^{* *}$ & $0.72^{* *}$ \\
\hline 6. Conflict & 0.427 & 0.198 & & & & & $\mathbf{X}$ & $-0.53^{* *}$ \\
\hline 7. Problem solving & 0.790 & 0.154 & & & & & & $\mathbf{X}$ \\
\hline
\end{tabular}

${ }^{\dagger} \mathrm{p}<.1 ;{ }^{*} \mathrm{p}<.05 ;{ }^{* *} \mathrm{p}<.01 ;{ }^{* * *} \mathrm{p}<.001$ 
Table 2.

Result of black-box regression analysis $(n=27)$

\begin{tabular}{|l|c|c|}
\hline & Beta & SE \\
\hline 2. Group size & 0.640 & 0.549 \\
\hline 3. Gender diversity & $7.610^{* *}$ & 2.179 \\
\hline 4. National diversity & $-4.697^{* * *}$ & 1.001 \\
\hline Constant & $13.151^{* * *}$ & 1.982 \\
\hline \multicolumn{2}{|c|}{0.532} \\
\hline Adj R & \multicolumn{2}{|c|}{$10.864^{* * *}$} \\
\hline \multicolumn{2}{|r|}{$\mathrm{F}<.1 ;{ }^{*} \mathrm{p}<.05 ;{ }^{* *} \mathrm{p}<.01 ;{ }^{* * *} \mathrm{p}<.001$} \\
\hline
\end{tabular}


Table 3.

Result of diversity explaining the process variable of problem solving $(n=27)$

\begin{tabular}{|l|c|c|}
\hline & Beta & SE \\
\hline 3. Gender diversity & 0.184 & 0.183 \\
\hline 4. National diversity & -0.139 & 0.084 \\
\hline Constant & $0.807^{* * *}$ & 0.043 \\
\hline \multicolumn{2}{|c|}{0.054} \\
\hline \multicolumn{2}{|c|}{ Adj R } & \multicolumn{2}{|c|}{1.737} \\
\hline \multicolumn{2}{|r|}{$\mathrm{F}$} & ${ }^{\dagger} \mathrm{p}<.1 ;{ }^{*} \mathrm{p}<.05 ;{ }^{* *} \mathrm{p}<.01 ;{ }^{* * *} \mathrm{p}<.001$
\end{tabular}


Table 4.

Result of process variables explaining performance $(n=27)$

\begin{tabular}{|l|c|c|}
\hline & Beta & SE \\
\hline 2. Group size & -0.011 & 0.047 \\
\hline 6. Conflict & 4.897 & 2.873 \\
\hline 7. Problem solving & $9.927 *$ & 3.591 \\
\hline Constant & 3.795 & 4.198 \\
\hline \multicolumn{2}{|c|}{0.172} \\
\hline \multicolumn{2}{|r|}{ Adj R } & \multicolumn{2}{|c|}{$2.800^{\dagger}$} \\
\hline \multicolumn{2}{|r|}{$\mathrm{F}$} & \multicolumn{2}{|c|}{} \\
\hline \multicolumn{2}{|r|}{$\mathrm{p}<.1{ }^{*} \mathrm{p}<.05 ;{ }^{* *} \mathrm{p}<.01 ;{ }^{* * *} \mathrm{p}<.001$}
\end{tabular}

